in this case. It is well known that histopathological changes in the liver damage of infants and children given total parenteral nutrition range from mild cholestasis to fibrosis or cirrhosis. ${ }^{6}$ Cholestasis may produce activation of Kupffer cells, stimulating proliferation and fibrogenesis of lipocytes which then show the structural characteristics of myofibroblasts, ${ }^{7}$ as found in our patients.

Proliferating blasts in transient abnormal myelopoiesis are known often to express antigens characteristic of megakaryocytes. ${ }^{8}$ Acute megakaryoblastic leukaemia (AMKL) is often accompanied by myelofibrosis with a marked increase in reticulin fibres. Although mechanisms of myelofibrosis are yet to be determined in AMKL, cytokines such as transforming growth factor $\beta,{ }^{9}$ as well as platelet derived growth factor, ${ }^{10}$ secreted from megakaryocytes can promote fibrogenesis. Both infants described here showed slight increases in reticulin fibre, a feature not described in previous reports of infants with Down syndrome, transient abnormal myelopoiesis, and hepatic fibrosis. ${ }^{1}$ This change might reflect an excessive release of cytokines or growth factors from proliferating haematopoietic cells, but not necessarily from megakaryocytes, since these were not increased.

1 Miyauchi J, Ito Y, Kawano T, Tsunematsu Y, Shimizu K Unusual liver fibrosis accompanying transient myeloproliferative disorder in Down's syndrome: a report of four autopsy cases and proposal of a hypothesis. Blood 1992;80:1521-7.

2 Miller JM, Sherrill JG, Hathaway WE. Thrombocythemia in myeloproliferative disorder of Down's syndrome. Pediatrics 1967;40:847-50.

3 Nakagawa T, Nishida H, Arai T, Yamada T, Fukuda $M$ Sakamoto S. Hyperviscosity syndrome with transient abSakamoto $S$. Hyperviscosity syndrome with transient abnormal my

4 Becroft DMO, Zwi LJ. Perinatal visceral fibrosis accompanying the megakaryoblastic leukemoid reaction of Down syndrome. Pediatr Pathol 1990;10:397-406.

5 Tsao M-S. Hepatic sinusoidal fibrosis in agnogenic myeloid metaplasia. Am f Clin Pathol 1989;91:302-5.

6 Cohen C, Olsen MM. Pediatric total parenteral nutrition liver pathology. Arch Pathol Lab Med 1981;105:152-6.

7 Friedman SL. The cellular basis of hepatic fibrosis: mech anism and treatment strategies. $N$ Engl $\mathcal{F}$ Med 1993;328: 1828-35.

8 Coulombel L, Derycke M, Villeval JL, Leonald C, BretonGorius J, Vial M. Characterization of the blast cell population in two neonates with Down's syndrome and transient myeloproliferative disorder. Br f Haematol 1987;66: 69-76.

9 Terui T, Niitsu Y, Mahara K, Fujisaki Y, Urushizaki Y, Mogi Y, et al. The production of transforming growth factor- $\beta$ in acute megakaryoblastic leukemia and its possible implications in myelofibrosis. Blood 1990;75: sible imp.

10 Castro-Malaspina H, Rabellino EM, Yen A, Nachman RL, Moore MAS. Human megakaryocyte stimulation of proliferation of bone marrow fibroblasts. Blood 1981;57 $781-7$.

\title{
Acute hepatic and renal failure caused by Pneumocystis carinii in patients with AIDS
}

\author{
R Boldorini, S Guzzetti, L Meroni, T Quirino, S Cristina, G Monga
}

\author{
Dipartimento \\ di Scienze Mediche, \\ II Facoltà di \\ Medicina e Chirurgia \\ di Novara-Università \\ di Torino, Italy \\ R Boldorini \\ S Cristina \\ G Monga \\ Istituto di \\ Scienze Biomediche \\ "L Sacco", \\ Università di Milano, \\ Milan, Italy \\ V Cattedra di \\ Anatomia Patologica \\ S Guzzetti \\ Clinica Malattie \\ Infettive \\ L Meroni \\ I Divisione \\ di Malattie Infettive \\ T Quirino \\ Correspondence to: \\ Dr Guido Monga, \\ Dipartimento di \\ Scienze Mediche-Via \\ Novara, Italy. \\ Accepted for publication \\ 13 March 1995
}

\begin{abstract}
Clinical and pathological findings are described in two AIDS patients with Pneumocystis carinii infection who received prophylactic treatment with nebulised pentamidine and developed unusual hepatic and renal failure. Histological examination showed clumps of $P$ carinii massively obstructing hepatic sinuses and portal vessels in the first patient, and merular and intertubular capillaries in the second. These findings could explain the unusual clinical features, characterised by acute hepatic and renal failure.

(f Clin Pathol 1995;48:975-978)
\end{abstract}

Keywords: AIDS, Pneumocystis carinii, hepatic failure, renal failure.

Disseminated pneumocystosis in patients with acquired immunodeficiency syndrome (AIDS) is reported with increasing frequency, ${ }^{1}$ but it appears still to be rare despite the high frequency of Pneumocystis carinii pneumonia. ${ }^{2} \mathrm{Ex}-$ trapulmonary localisation of pneumocystis has been reported in necropsy series and in single case reports, with the involvement of spleen, lymph nodes, bone marrow, liver, thyroid, gut, adrenal gland, skin, and brain. ${ }^{3}$

Extrapulmonary pneumocystosis can give rise to unusual clinical presentation, including thyroiditis, otitis media with mastoiditis, cutaneous lesions, "acute abdomen", hepatitis and choroiditis, and lymph node enlargement. ${ }^{4}$ To our knowledge, there are no well documented reports of renal failure due to $P$ carinii. Only a few cases of hepatic failure have been described on the basis of the clinical findings, ${ }^{5}$ but without morphological evidence of the type and severity of liver damage.

We describe the clinical and pathological features of two cases of disseminated pneumocystosis in AIDS patients who were given prophylactic treatment with nebulised pentamidine and developed hepatic and renal failure because of severe liver and kidney damage.

\section{Methods}

Necropsy examinations were performed 12 hours (patient 1) and 17 hours (patient 2) 


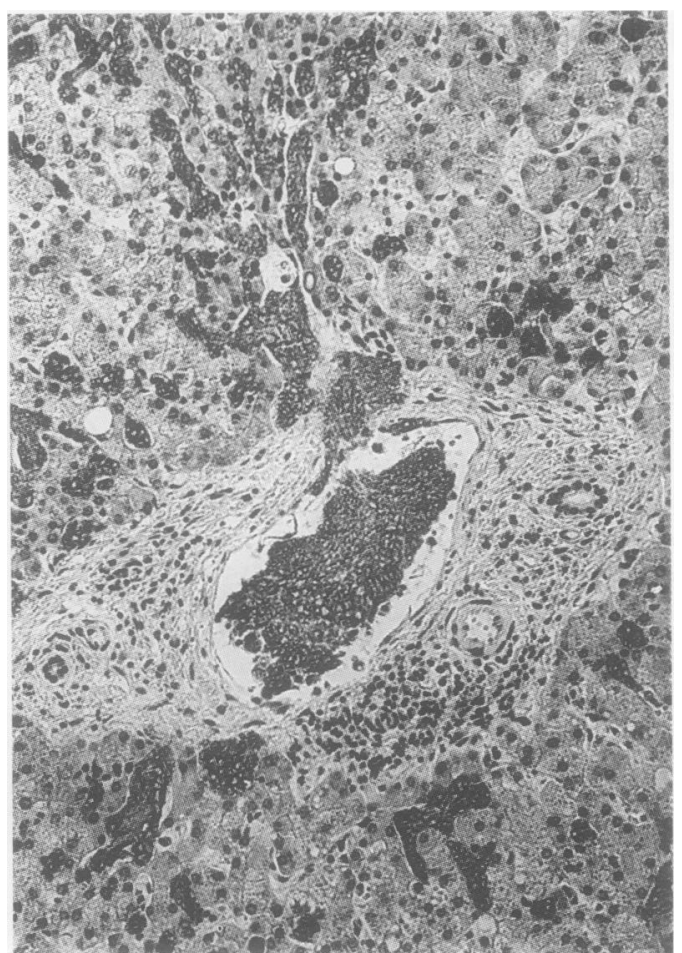

Figure 1 Immunocytochemical demonstration of Pneumocystis carinii cysts filling portal and sinusoidal vessels in case 1. Immunoperoxidase, haematoxylin counterstaining, $\times 250$.

after death. Tissues were fixed in formalin, embedded in paraffin, and stained with haematoxylin and eosin, periodic acid-Schiff, Grocott-Gomori, and Giemsa. Immunohistochemistry was performed on paraffin embedded tissues with the monoclonal antibody $3 F 6$ against the $82 \mathrm{kDa}$ component of the $P$ carinii cyst wall (Dakopatts, dilution 1 in 40).

\section{Case reports}

PATIENT 1

A 28 year old female drug addict with AIDS was admitted to hospital in July 1989 because of cough and dyspnoea. Pneumocystis pneumonia was diagnosed by bronchoalveolar lavage and treated with a course of trimethoprimsulphamethoxazole which produced clinical and radiological resolution. After recovery, prophylaxis with nebulised pentamidine was begun. In August 1991 she was again admitted to hospital, with a clinical diagnosis of severe hepatic failure, ascites, lower limb oedema, and splenomegaly. Laboratory data included the following abnormal values: alanine aminotransferase $170 \mathrm{IU} / 1, \gamma$-glutamyl aminotransferase $(\gamma-G T) 174 \mathrm{IU} / \mathrm{l}$, alkaline phosphatase $1439 \mathrm{IU} / \mathrm{l}$, lactate dehydrogenase $1158 \mathrm{IU} / \mathrm{l}$, proteins $4 \mathrm{~g} / 100 \mathrm{ml}$, albumin $1 \mathrm{~g} / 100 \mathrm{ml}$. The CD $4+$ cell count was 6 cells $/ \mu \mathrm{l}, \mathrm{CD} 8+$ 129 cells $/ \mathrm{ml}$, with a T-helper/T-suppressor cell ratio of $0 \cdot 05$. A chest $x$ ray revealed mild diffuse interstitial infiltrates in both lungs. Paracentesis was immediately performed to resolve the abdominal symptoms. The patient died 24 hours later, before beginning specific treatment for hepatic failure.
PATIENT 2

A 34 year old man with AIDS was admitted to hospital in September 1992 with a four month history of persistent fever and weight loss. He had been on primary prophylaxis with nebulised pentamidine since 1991. Laboratory data on admission were: haemoglobin $7 \cdot 4 \mathrm{~g} /$ $100 \mathrm{ml}$; white blood count $2200 / \mathrm{mm}^{3}$; platelet count $194000 / \mathrm{mm}^{3}$; alkaline phosphatase $0.417 \mathrm{IU} / 1 ; \gamma$-GT $187 \mathrm{IU} / \mathrm{l}$; lactate dehydrogenase $732 \mathrm{IU} / 1$. CD4 + cell count was 3 cells/ $\mathrm{ml}, \mathrm{CD} 8+40$ cells $/ \mu \mathrm{l}$, with a T-helper/T-suppressor cell ratio of $0 \cdot 01$. A chest $x$ ray showed bilateral pulmonary interstitial infiltrates. Blood cultures revealed the presence of $\mathrm{Myco}$ bacterium avium infection. Specific antibiotic therapy did not produce complete remission of the symptoms. Abdominal ultrasonography revealed many nodules in the liver, spleen, and kidney. A bone marrow biopsy disclosed many forms of $P$ carinii in the small vessels. The patient was therefore given therapy with intravenous pentamidine $(200 \mathrm{mg} /$ day $)$. Two days later, renal failure occurred suddenly, with increasing azotaemia (plasma urea increasing from 5.0 to $21.6 \mathrm{mmol} / \mathrm{l}$ ) and creatininaemia (plasma creatinine increasing from 133 to 840 $\mu \mathrm{mol} / \mathrm{l})$. The patient died 48 hours later.

\section{Pathological findings}

\section{PATIENT 1}

The lungs (combined weight: $1500 \mathrm{~g}$ ) were red-grey and diffusely indurated. The pleura contained some miliary nodules. Forms of $P$ carinii were observed in the alveoli and septa

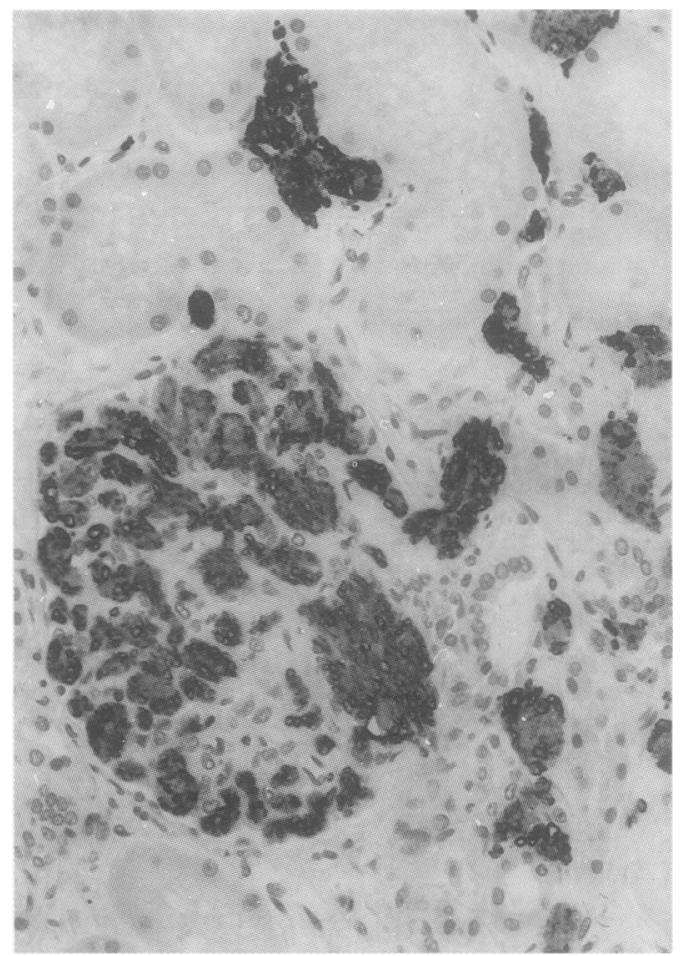

Figure 2 Massive embolisation of glomerular and intertubular capillary vessels by Pneumocystis carinii cysts, seen clearly after staining with specific pneumocystis antiserum in case 2. Immunoperoxidase, haematoxylin counterstaining, $\times 400$. 
and diffusely in lymphatics and blood vessels. Granulomas and calcifications, some immunohistochemically positive for pneumocystis, were present. Extensive alveolar and septal necrosis replaced by confluent masses of foamy material containing aggregates of pneumocystis were found.

Liver and spleen were enlarged (2000 and $1300 \mathrm{~g}$ ); tan nodules of various sizes (from a few millimetres to $2-3 \mathrm{~cm}$ ), occasionally calcified, were observed in the spleen. The liver was redgrey and small, scattered white nodules were present on the cut surface. Histological examination revealed portal vessels and sinuses massively dilated and filled by pneumocystis cysts (fig 1) which often appeared calcified. On occasion, small necrotic areas were also found in the lobuli. Portal and lobular inflammation was scarce. Occasional pneumocystis emboli were found in the vessels of the adrenal glands, lymph nodes, lamina propria, and submucosa of gut, bone marrow, kidney, and meninges.

\section{PATIENT 2}

The lungs were enlarged (combined weight $2030 \mathrm{~g}$ ), with a dry, reddish-grey cut surface and with little oedema of the lower lobes. Pneumocystis forms were found diffusely in septal, peribronchiolar, and bronchial vessels, but only occasionally in the alveolar spaces. Degenerative forms of pneumocystis, with calcification and necrosis, were seen extensively . Liver and spleen were enlarged (1900 and $650 \mathrm{~g}$ ), showing many yellowish-white nodules containing caseous-like material when cut. Scattered pneumocystis emboli were found in the hepatic portal vessels and sinuses. The spleen had a large amount of pneumocystis replacing the parenchyma and a few granulomas with macrophages containing $\mathrm{Myco}$ bacterium avium-intracellulare. Nodules were also seen in the pancreas, heart, adrenal glands, and thyroid. In all these organs, many pneumocystis cysts embolised small vessels, often with local infarction. The kidney (combined weight $420 \mathrm{~g}$ ) was the organ most involved. Miliary nodules were diffusely distributed throughout the surfaces of both kidneys, especially on the cortex. Almost all the glomeruli showed capillary lumens widened and filled with pneumocystis (fig 2); glomerular cellularity was usual and inflammatory cell were absent. In addition, pneumocystis cysts were found extensively in the intertubular capillaries and in the venules. Large parenchymal areas had ischaemic necrosis and were filled with aggregates of pneumocystis cysts.

\section{Discussion}

Even in patients with AIDS, $P$ carinii is rarely suspected to be the causative agent responsible for infection outside the lungs, probably because extrapulmonary pneumocystosis is frequently asymptomatic and its occurrence is considered unlikely. Patient 1 developed severe ascites and hepatic failure. Although she had had a previous pneumocystis pneumonia infection two years before admission, ex- trapulmonary localisation of pneumocystis was not suspected clinically. Mathews et al described three cases of severe ascites and hepatic failure caused by pneumocystis. However, in these cases the physicians were alerted to the possibility of extrapulmonary infection by the presence of a typical choroidal lesion suggestive of extrapulmonary localisation of pneumocystis, which was absent in our patient.

The liver involvement is common in disseminated pneumocystosis, but only a few reports describe hepatic disease. ${ }^{57}$ Acute hepatic failure is occasionally reported as acute hepatitis, ${ }^{5}$ only on the grounds of clinical data. The extensive liver damage in our patient supplies the morphological evidence of the hepatic failure. A severe deficit of protein (especially of albumin) was present in our patient. The mechanism of hypoalbuminaemia and hepatic dysfunction caused by massive involvement of the liver by pneumocystis is unknown. Mathews et $a l^{6}$ did not observe any external loss of albumin in their three cases, and suggested that the hypoalbuminaemia might be caused by increased turnover associated with a decrease of albumin synthesis. Our case provides some evidence that the hypoalbuminaemia might be related to direct liver cell damage evolving focally in tissue necrosis, and that ascites could be due to both hypoalbuminaemia and diffuse mechanical obstruction of the sinuses by pneumocystis emboli.

Renal involvement was the prevailing feature both clinically and pathologically in patient 2 . Renal involvement with pneumocystis is not particularly rare in disseminated pneumocystosis, ${ }^{89}$ especially in necropsy data, but to our knowledge this is the first clinical and necropsy report strongly suggesting that $P \mathrm{ca}$ rinii was the cause of acute renal failure. Indeed, pneumocystis aggregates were seen diffusely embolising glomerular capillaries and cortical and medullary vessels, with extensive areas of necrosis.

The reasons for dissemination of pneumocystis infection are still unknown. The usual confinement of the infection to pulmonary alveoli may indicate that local factors could be particularly beneficial for the growth of the organism, but the recently reported cases of extrapulmonary localisation of pneumocystis confirm that the organism is able to survive in both aerobic and anaerobic environments. A possible explanation of the increased frequency of disseminated pneumocystosis is the existence of different $P$ carinii strains (that is, selected by treatment). ${ }^{4}$ Another important mechanism in disseminated pneumocystosis could be the failure of nebulised pentamidine to control the pulmonary infection and the low systemic levels of pentamidine. ${ }^{1}$ In fact, only rare cases of disseminated pneumocystosis were reported before the introduction of nebulised pentamidine ${ }^{10}$ and both our patients received such treatment.

In conclusion, our data provide further evidence that $P$ carinii infection may be responsible for the unfavourable outcome in AIDS patients, not only because of pulmonary damage, but also because of massive localisation at other 
sites. Clinicians must be aware of this possibility, which should be suspected in AIDS patients with unusual clinical findings.

1 Northfelt DW, Clement MJ, Safrin S. Extrapulmonary deficiency virus infection. Medicine 1990;69:392-8.

2 Hopewell PC. Pneumocystis carinii pneumonia: diagnosis. F Infect Dis 1988;6:1115-23.

3 Watts CJ, Chandler FW. Evolving concepts of infection with Pneumocystis carinii. In: Rosen PP, Fechner RE, eds. Pathology annual part 1. Norwalk, San Mateo: Appleton and Lange, 1991: 93-138.

4 Raviglione MC Extrapulmonary pneumocystosis: the first 50 cases. Rev Infect Dis 1990;12:380-6.

5 Poblete RB, Rodriguez K, Foust RT, Reddy RK, Saldana MJ. Pneumocystis carinii hepatitis in the acquired immune deficiency syndrome (AIDS). Ann Intern Med 1989;110: 737-8.

6 Mathews WC, Bozzette SA, Harrity S, Meurer W, Viesca T. Pneumocystis carinii peritonitis. Antemortem confirmation of disseminated pneumocystosis by cytologic firmation of disseminated pneumocystosis by cytologic 867-9.

7 Sachs JR, Greenfield SM, Sohn M, Turner JL. Disseminated Pneumocystis carinii infection with hepatic involvement in a patient with the acquired immune deficiency syndrome. Am f Gastroenterol 1991;86:82-5.

8 Quirino T, Capetti A, Boldorini R, Iemoli E, Almaviva M, Vigevani GM. Pneumocystose disséminée: 3 cas chez des malades séropositifs pour le VIH [letter]. Presse Med 1993; 22:1013.

9 Coker RJ, Clark D, Claydon EL, Gorpels M, Ainsworth JB, Lucas SB, et al. Disseminated Pneumocystis carinii in AIDS. 7 Clin Pathol 1991;44:820-3.

10 Awen CF, Baltzan MA. Systemic dissemination of Pneumocystis carinii pneumonia. Can Med Assoc f 1971;104: 809-12. 